IJMMS 32:5 (2002) 319-324

PII. S0161171202007895

http://ijmms.hindawi.com

(c) Hindawi Publishing Corp.

\title{
SUFFICIENT CONDITIONS FOR STARLIKENESS ASSOCIATED WITH PARABOLIC REGION
}

\author{
V. RAVICHANDRAN, A. GANGADHARAN, and T. N. SHANMUGAM
}

Received 15 June 2001

\begin{abstract}
An analytic function $f(z)=z+a_{n+1} z^{n+1}+\cdots$, defined on the unit disk $\triangle=\{z:|z|<1\}$, is in the class $S_{p}$ if $z f^{\prime}(z) / f(z)$ is in the parabolic region $\operatorname{Re} w>|w-1|$. This class is closely related to the class of uniformly convex functions. Sufficient conditions for function to be in $S_{p}$ are obtained. In particular, we find condition on $\lambda$ such that the function $f(z)$, satisfying $(1-\alpha)(f(z) / z)^{\mu}+\alpha f^{\prime}(z)(f(z) / z)^{\mu-1} \prec 1+\lambda z$, is in $S_{p}$.
\end{abstract}

2000 Mathematics Subject Classification: 30C45.

1. Introduction. Let $\mathscr{A}_{n}$ be the family of analytic functions $f(z)=z+a_{n+1} z^{n+1}+$ $\cdots$ in the unit disk $\triangle=\{z:|z|<1\}$, and let $\mathscr{A}_{1}=\mathscr{A}$. For $0 \leq \alpha<1$, let $S^{*}(\alpha)$ and $C(\alpha)$ denote the subclasses of $\mathscr{A}$ of starlike functions and convex functions of order $\alpha$, respectively; for $\alpha=0, S^{*}(0)=S^{*}$, the class of starlike functions in $\triangle$. The function $f \in \mathscr{A}$ is uniformly convex (starlike) if, for every circular arc $\gamma$ contained in $\triangle$ with center $\zeta \in \triangle$, the image arc $f(\gamma)$ is convex (starlike with respect to $f(\zeta)$ ). The class of all uniformly convex functions denoted by UCV was introduced by Goodman [1] in 1991. Rønning [5] and Ma and Minda [2] independently proved that $f \in \mathrm{UCV}$ if and only if

$$
\operatorname{Re}\left\{1+\frac{z f^{\prime \prime}(z)}{f^{\prime}(z)}\right\}>\left|\frac{z f^{\prime \prime}(z)}{f^{\prime}(z)}\right|, \quad z \in \triangle
$$

Further, Rønning [5] defined the class $S_{p}$ of functions $f \in \mathscr{A}$ for which

$$
\operatorname{Re}\left\{\frac{z f^{\prime}(z)}{f(z)}\right\}>\left|\frac{z f^{\prime}(z)}{f(z)}-1\right|
$$

holds for all $z \in \triangle$. It can be observed that $f \in \mathrm{UCV}$ if and only if $z f^{\prime} \in S_{p}$. Let $\Omega=\{w:|w-1|<\operatorname{Re} w\}$. It follows that $f \in \mathrm{UCV}$ or $S_{p}$ are equivalent to saying that $1+z f^{\prime \prime}(z) / f^{\prime}(z)$ or $z f^{\prime}(z) / f(z)$ are in $\Omega$, respectively. Note that $\Omega$ is a parabolic region symmetric with respect to the real axis and $(1 / 2,0)$ as its vertex. The function $k(z)$, with $k(0)=k^{\prime}(0)-1=0$ and

$$
1+\frac{z k^{\prime \prime}(z)}{k^{\prime}(z)}=1+\frac{2}{\pi^{2}}\left[\log \left(\frac{1+\sqrt{z}}{1-\sqrt{z}}\right)\right]^{2},
$$

is an example of function in UCV. 
Ponnusamy and Singh [4] obtained bounds on $\lambda$ such that the Alexander transform of $f \in \mathscr{A}$, satisfying $f^{\prime} \prec 1+\lambda z$, is uniformly convex. We extend their result in two directions. Specifically, we find condition on $\lambda$ such that the function $f(z)$, satisfying

$$
\begin{gathered}
(1-\alpha)\left(\frac{f(z)}{z}\right)^{\mu}+\alpha f^{\prime}(z)\left(\frac{f(z)}{z}\right)^{\mu-1} \prec 1+\lambda z, \\
\left|\alpha z f^{\prime \prime}(z)+f^{\prime}(z)-1\right|<\lambda,
\end{gathered}
$$

is in $S_{p}$.

Let $a>1 / 2$ and let $R_{a}=\min \{|w-a|:|w-1|=\operatorname{Re} w\}$. A simple computation gives

$$
R_{a}= \begin{cases}a-\frac{1}{2} & \text { if } \frac{1}{2}<a \leq \frac{3}{2}, \\ \sqrt{2 a-2} & \text { if } a \geq \frac{3}{2} .\end{cases}
$$

Now, $D\left(a, R_{a}\right)=\left\{w:|w-a|<R_{a}\right\}$ is the largest disk centered at $a$ which lies inside $\Omega$. If we restrict the value of $a$ by $3 / 4<a<3$, then the disk will contain the point 1 .

LEMMA 1.1 [6]. Let $f \in \mathscr{A}$. If, for any $a, 3 / 4<a<3$,

$$
\left|\frac{z f^{\prime}(z)}{f(z)}-a\right|<R_{a}, \quad z \in U
$$

then $f \in S_{p}$.

Also, we need the following result.

LEMMA 1.2 [3]. Let $h(z)$ be convex and $\gamma \neq 0$, Re $\gamma \geq 0$. If $p(z)=a+p_{n} z^{n}+\cdots$, $n \geq 2$, is analytic in $\triangle$ and

$$
p(z)+\frac{z p^{\prime}(z)}{\gamma} \prec h(z), \quad h(0)=p(0),
$$

then

$$
p(z) \prec \frac{\gamma}{n} z^{-\gamma / n} \int_{0}^{z} h(t) t^{\gamma / n-1} d t .
$$

2. Main results. We begin with proving the following result.

THEOREM 2.1. Let $\mu>0, \alpha \geq 0$, and $0 \leq \beta<1$. Let $f \in A_{n}$ and

$$
0<\lambda \leq \frac{\alpha(\mu+\alpha n)(a-\beta-|1-a|)}{\mu[1+(a-\beta) \alpha+|(a-1) \alpha+1|]+\alpha n} .
$$

Then, for $a>(1+\beta) / 2$,

$$
(1-\alpha)\left(\frac{f(z)}{z}\right)^{\mu}+\alpha f^{\prime}(z)\left(\frac{f(z)}{z}\right)^{\mu-1} \prec 1+\lambda z
$$

implies

$$
\left|\frac{z f^{\prime}(z)}{f(z)}-a\right| \leq \frac{\lambda[\mu+\alpha n+\mu|(a-1) \alpha+1|]+\alpha|1-a|(\mu+\alpha n)}{\alpha(\mu+\alpha n-\lambda \mu)} \leq a-\beta,
$$

and $f \in S^{*}(\beta)$. 
Proof. Define the functions $Q(z)$ and $w(z)$ by

$$
Q(z)=\left(\frac{f(z)}{z}\right)^{\mu}, \quad w(z)=\frac{z f^{\prime}(z)}{f(z)}-a, \quad z \in \triangle
$$

Then, $Q(z)$ and $w(z)$ are analytic in $\triangle$, and $w(0)=1-a$. Clearly,

$$
\begin{gathered}
(1-\alpha) Q(z)+\alpha[w(z)+a] Q(z)=(1-\alpha)\left(\frac{f(z)}{z}\right)^{\mu}+\alpha \frac{z f^{\prime}(z)}{f(z)}\left(\frac{f(z)}{z}\right)^{\mu} \prec 1+\lambda z, \\
\frac{1}{\mu} \frac{z Q^{\prime}(z)}{Q(z)}+1=w(z)+a .
\end{gathered}
$$

This shows that

$$
Q(z)+\frac{\alpha}{\mu} z Q^{\prime}(z) \prec 1+\lambda z
$$

and hence, by Lemma 1.2, we have

$$
Q(z) \prec 1+\frac{\lambda \mu}{\mu+\alpha n} z, \quad z \in \triangle .
$$

Since

$$
\lambda \leq \frac{\alpha(\mu+\alpha n)(a-\beta-|1-a|)}{\mu[1+(a-\beta) \alpha+|(a-1) \alpha+1|]+\alpha n} \leq \frac{\mu+\alpha n}{\mu}
$$

and $a \geq(1+\beta) / 2$, we see that $\mu+\alpha n-\lambda \mu>0$.

Since

$$
\begin{aligned}
& \frac{z f^{\prime}(z)}{f(z)}-a=w(z) \\
& \quad=\frac{[(1-\alpha) Q(z)+\alpha Q(z)(w(z)+a)-1]-(Q(z)-1)[(a-1) \alpha+1]+\alpha(1-a)}{\alpha Q(z)},
\end{aligned}
$$

we have

$$
\begin{aligned}
\left|\frac{z f^{\prime}(z)}{f(z)}-a\right| & \leq \frac{\lambda+(\lambda \mu /(\mu+\alpha n))|(a-1) \alpha+1|+\alpha|1-a|}{\alpha(1-\lambda \mu /(\mu+\alpha n))} \\
& \leq \frac{\lambda[\mu+\alpha n+\mu|(a-1) \alpha+1|]+\alpha|1-a|(\mu+\alpha n)}{\alpha(\mu+\alpha n-\lambda \mu)} \\
& \leq a-\beta
\end{aligned}
$$

provided condition (2.1) is satisfied. This shows that $\operatorname{Re} z f^{\prime}(z) / f(z)>\beta$ and $f(z)$ is starlike of order $\beta$.

Note that to prove (2.10) it is enough to assume that $0<\lambda \leq(\mu+\alpha n) / \mu$. 
COROLLARY 2.2. If $f(z)=z+a_{n+1} z^{n+1}+\cdots$ is analytic in $\triangle$ and if

$$
\left|f^{\prime}(z)\left(\frac{f(z)}{z}\right)^{\mu-1}-1\right|<\lambda, \quad z \in \triangle,
$$

then, for $a>1 / 2$, we have

$$
\left|\frac{z f^{\prime}(z)}{f(z)}-a\right| \leq \frac{\lambda[\mu(a+1)+n]+(\mu+n)|1-a|}{\mu+n-\lambda \mu} \leq a
$$

provided $\mu>0$ and

$$
0<\lambda \leq \frac{(\mu+n)(a-|1-a|)}{\mu(1+2 a)+n} .
$$

When $\mu=1$, Corollary 2.2 reduces to the result by Ponnusamy and Singh [4].

THEOREM 2.3. Let $\lambda$ be defined by

$$
\lambda= \begin{cases}\frac{\alpha(\mu+\alpha n)(4 a-3)}{\mu[2+(2 a-1) \alpha+2|(a-1) \alpha+1|]+2 \alpha n} & \left(\frac{3}{4}<a \leq 1\right), \\ \frac{\alpha(\mu+\alpha n)}{\mu[\alpha(4 a-1)+2]+2 \alpha n} & \left(1 \leq a \leq \frac{3}{2}\right), \\ \frac{\alpha(\mu+\alpha n)(1-a+\sqrt{2 a-2})}{\alpha n+\mu[2+\alpha(a-1)+\alpha \sqrt{2 a-2}]} & \left(\frac{3}{2} \leq a<3\right) .\end{cases}
$$

If $f \in A_{n}$ satisfies

$$
(1-\alpha)\left(\frac{f(z)}{z}\right)^{\mu}+\alpha f^{\prime}(z)\left(\frac{f(z)}{z}\right)^{\mu-1} \prec 1+\lambda z,
$$

then $f \in S_{p}$.

It should be noted that if $3 / 4<a \leq 3 / 2$, then the condition on $\lambda$ in Theorem 2.3 reduces to the condition in Theorem 2.1. Hence, with the same condition as in Theorem 2.1 (with $\beta=1 / 2$ ), we get a stronger conclusion that $f \in S_{p}$.

Proof. We first verify that $\lambda$ defined in Theorem 2.3 satisfies the condition $0<$ $\lambda \leq(\mu+\alpha n) / \mu$. This condition is equivalent to

$$
0 \leq \begin{cases}\mu[\alpha(2 a-1)+1] & \left(1 \leq a \leq \frac{3}{2}\right), \\ 2 \mu+\alpha n & \left(\frac{3}{4} \leq a \leq 1,(a-1) \alpha+1 \geq 0\right), \\ 2 \mu \alpha(1-a)+\alpha n & \left(\frac{3}{4} \leq a \leq 1,(a-1) \alpha+1 \leq 0\right), \\ \alpha n+2 \mu[\alpha(a-1)+1] & \left(\frac{3}{2} \leq a<3\right) .\end{cases}
$$

The above inequality is obviously correct. Let

$$
R_{a}=\frac{\lambda[\mu+\alpha n+\mu|(a-1) \alpha+1|]+\alpha|1-a|(\mu+\alpha n)}{\alpha(\mu+\alpha n-\lambda \mu)} .
$$


Then, a computation shows that

$$
R_{a}= \begin{cases}a-\frac{1}{2} & \left(\frac{3}{4}<a \leq \frac{3}{2}\right), \\ \sqrt{2 a-2} & \left(\frac{3}{2} \leq a<3\right) .\end{cases}
$$

Then, from the proof of Theorem 2.1, we have

$$
\left|\frac{z f^{\prime}(z)}{f(z)}-a\right| \leq R_{a} .
$$

Using Lemma 1.1, we have the desired result.

This result for $\mu=1$ and $\alpha=1$ is obtained in Ponnusamy and Singh [4].

TheOrem 2.4. Suppose $\alpha \in \mathbb{C}, a>1 / 2$, and $\lambda \in \mathbb{R}$ satisfy

$$
0<\lambda \leq \frac{|1+n \alpha|(\mu+n)(a-|1-a|)}{\mu(1+2 a)+n} .
$$

If

$$
\left(\frac{f(z)}{z}\right)^{\mu-1}\left\{\alpha(\mu-1) \frac{z\left[f^{\prime}(z)\right]^{2}}{f(z)}+\alpha z f^{\prime \prime}(z)+(1+(1-\mu) \alpha) f^{\prime}(z)\right\} \prec 1+\lambda z, \quad z \in \triangle,
$$

then

$$
\left|\frac{z f^{\prime}(z)}{f(z)}-a\right| \leq \frac{\lambda_{1}[n+\mu(a+1)]+(\mu+n)|1-a|}{\mu\left(1-\lambda_{1}\right)+n},
$$

where $\lambda_{1}=\lambda /|1+n \alpha|$.

Proof. Let $p(z)=f^{\prime}(z)(f(z) / z)^{\mu-1}, z \in \triangle$. Then, $p(z)$ is analytic in $\triangle$ and

$$
z p^{\prime}(z)=\left(\frac{f(z)}{z}\right)^{\mu-1}\left\{z f^{\prime \prime}(z)+(\mu-1)\left(\frac{z f^{\prime}(z)}{f(z)}-1\right) f^{\prime}(z)\right\} \text {. }
$$

This shows that

$$
p(z)+\alpha z p^{\prime}(z) \prec 1+\lambda z
$$

and hence, by Lemma 1.2,

$$
p(z) \prec 1+\frac{\lambda}{1+n \alpha} z \prec 1+\frac{\lambda}{|1+n \alpha|} z .
$$

The result now follows from Theorem 2.1 where $\lambda_{1}=\lambda /|1+n \alpha|$.

Corollary 2.5. Suppose that $\alpha \in \mathbb{C}, a>1 / 2$, and $\lambda \in \mathbb{R}$ satisfy

$$
0<\lambda \leq \frac{|1+n \alpha|(1+n)(a-|1-a|)}{1+2 a+n} .
$$

If $f \in \mathscr{A}$ satisfies

$$
\left|\alpha z f^{\prime \prime}(z)+f^{\prime}(z)-1\right|<\lambda, \quad z \in \triangle
$$

then

$$
\left|\frac{z f^{\prime}(z)}{f(z)}-a\right| \leq \frac{\lambda_{1}[n+a+1]+(1+n)|1-a|}{1-\lambda_{1}+n},
$$

where $\lambda_{1}=\lambda /|1+n \alpha|$. 
The result follows from Theorem 2.4 when $a=n=1$ and is obtained in [4].

THEOREM 2.6. Let $\lambda$ be defined by

$$
\lambda= \begin{cases}\frac{|1+n \alpha|(1+n)(4 a-3)}{4 a+2 n+1} & \left(\frac{3}{4}<a \leq 1\right), \\ \frac{|1+n \alpha|(1+n)}{4 a+2 n+1} & \left(1<a \leq \frac{3}{2}\right), \\ \frac{|1+n \alpha|(1+n)(\sqrt{2 a-2}+1-a)}{n+1+a+\sqrt{2 a-2}} & \left(\frac{3}{2} \leq a \leq 3\right) .\end{cases}
$$

If $\left|\alpha z f^{\prime \prime}(z)+f^{\prime}(z)-1\right|<\lambda$, then $f \in S_{p}$.

Proof. From the definition of $\lambda$, it is clear that

$$
\frac{\lambda_{1}[n+a+1]+(1+n)|1-a|}{1-\lambda_{1}+n}= \begin{cases}a-\frac{1}{2} & \left(\frac{3}{4}<a \leq \frac{3}{2}\right), \\ \sqrt{2 a-2} & \left(\frac{3}{2} \leq a<3\right),\end{cases}
$$

where $\lambda_{1}=\lambda /|1+n \alpha|$. Since

$$
0<\lambda \leq \frac{|1+n \alpha|(1+n)(a-|1-a|)}{2 a+n+1},
$$

the result follows from Corollary 2.5.

\section{REFERENCES}

[1] A. W. Goodman, On uniformly convex functions, Ann. Polon. Math. 56 (1991), no. 1, 87-92.

[2] W. C. Ma and D. Minda, Uniformly convex functions, Ann. Polon. Math. 57 (1992), no. 2, $165-175$.

[3] S. S. Miller and P. T. Mocanu, Differential subordinations and univalent functions, Michigan Math. J. 28 (1981), no. 2, 157-172.

[4] S. Ponnusamy and V. Singh, Criteria for strongly starlike functions, Complex Variables Theory Appl. 34 (1997), no. 3, 267-291.

[5] F. Rønning, Uniformly convex functions and a corresponding class of starlike functions, Proc. Amer. Math. Soc. 118 (1993), no. 1, 189-196.

[6] T. N. Shanmugam and V. Ravichandran, Certain properties of uniformly convex functions, Computational Methods and Function Theory (Penang, Malaysia, 1994) (R. M. Ali, St. Ruscheweyh, and E. B. Saff, eds.), Ser. Approx. Decompos., vol. 5, World Scientific Publishing, New Jersey, 1995, pp. 319-324.

V. RaVichandran: Department of Mathematics and Computer Applications, SRi VENKATESWARA COLLEGE OF ENGINEERING, SRIPERUMBUdUR 602 105, INDIA

E-mail address: vravi@svce.ac. in

A. Gangadharan: Department of Mathematics and Computer Applications, SRi VENKATESWARA COLLEge OF ENGINEERING, SRIPERUMBUdur 602 105, INDIA

T. N. Shanmugam: Department of Mathematics, ANNA University, Madras 600 025, INDIA

E-mail address: shan@annauniv.edu 


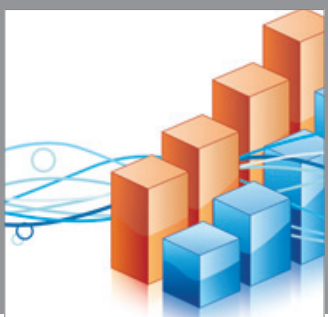

Advances in

Operations Research

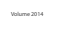

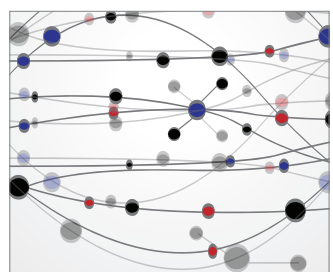

\section{The Scientific} World Journal
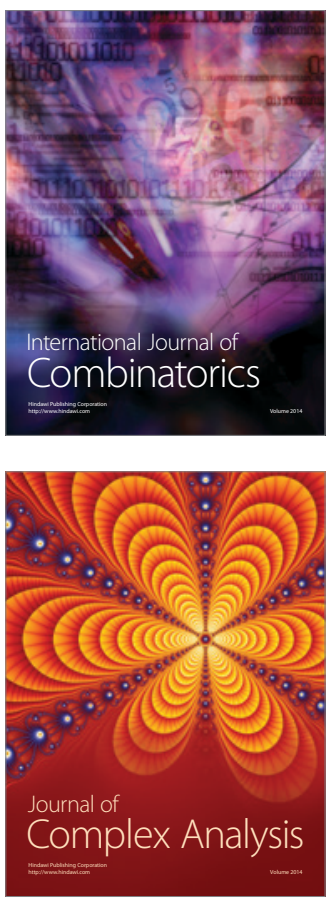

International Journal of

Mathematics and

Mathematical

Sciences
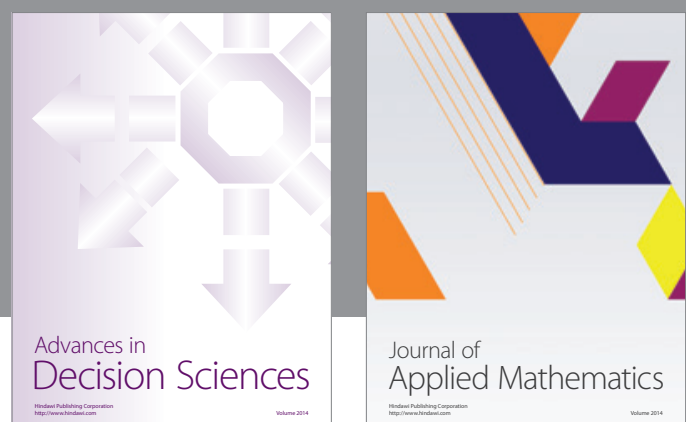

Journal of

Applied Mathematics
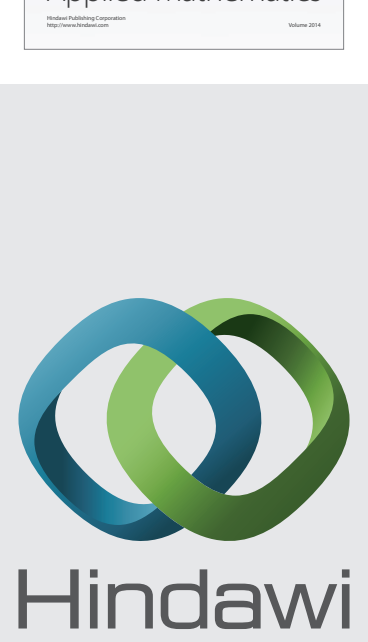

Submit your manuscripts at http://www.hindawi.com
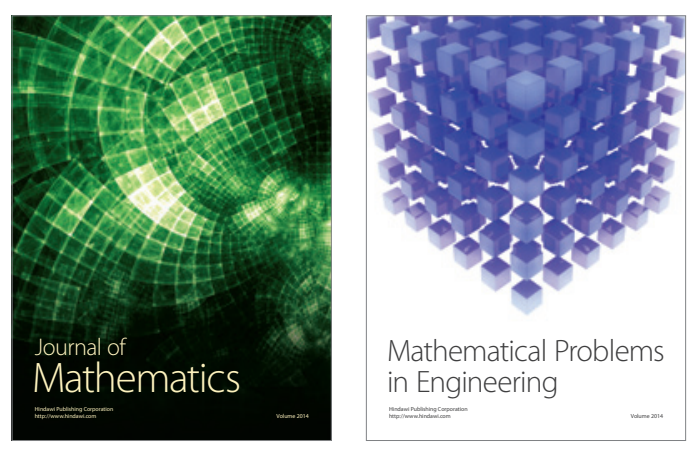

Mathematical Problems in Engineering
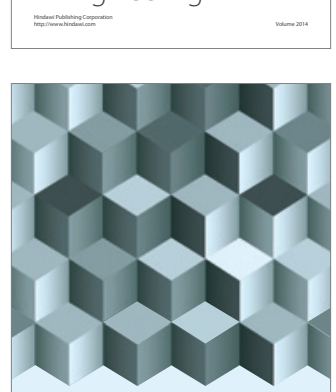

Journal of

Function Spaces
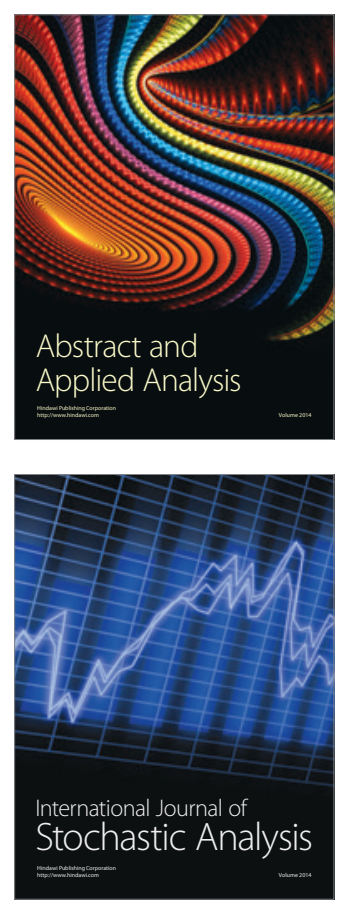

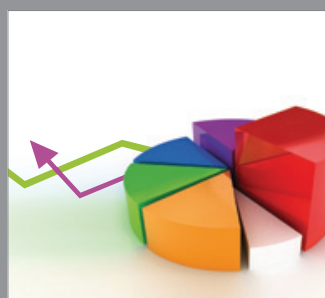

ournal of

Probability and Statistics

Promensencen
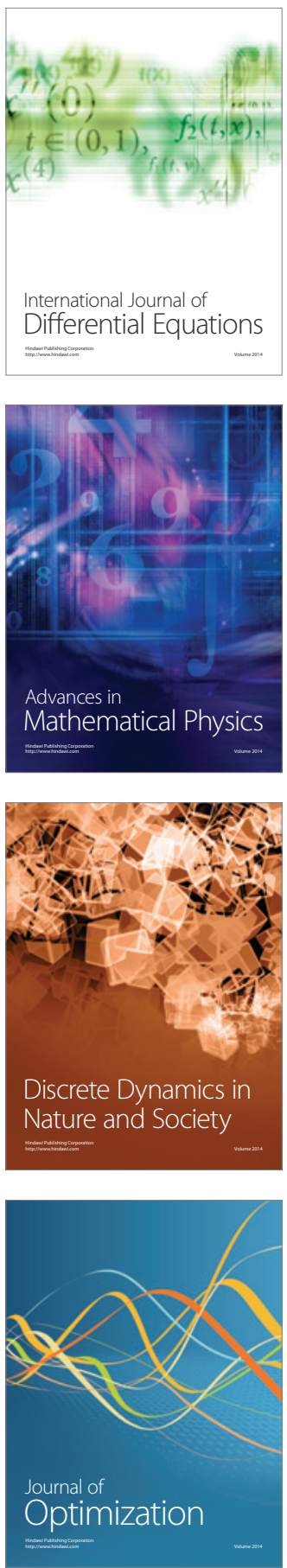